\title{
In response to the article of Dr. Nadia Peparini, "Nonclosure techniques and ductal decompression: a winning combination against the development of pancreatic fistula after distal pancreatectomy"
}

\author{
Hirohisa Kitagawa $\cdot$ Tetsuo Ohta
}

Received: 8 December 2008 / Accepted: 8 December 2008/Published online: 10 March 2009

(C) Springer 2009

In response,

We appreciate the comments and interest from Peparini and Chirletti regarding our article on strategies for preventing pancreatic fistula after distal pancreatectomy.

We believe pancreatic fistula after distal pancreatectomy can be divided into two categories. One is from the main pancreatic duct, and the other is from the interrupted smaller branches of the pancreatic duct. Both of these require measures to be taken against them. For preventing the fistula from the main pancreatic duct, we perform a reliable ligation of the main pancreatic duct and stenting of the remnant pancreatic duct. On the other hand, for preventing the fistula from the interrupted smaller branches, we sealed the surface of the cut end with saline-coupled bipolar electrocautery. Especially for the reliable and stable ligation of the main pancreatic duct, blood supply to the pancreatic parenchyma around the ligated portion is a very important factor for reducing pancreatic fistula after pancreaticoduodenectomy [1]. The blood supply is reduced by closing the cut end of the pancreas, which causes the pancreatic parenchyma to be squeezed by strings or staples used in the procedure. The

This reply refers to the letter that can be found at doi:10.1007/s00534-009-0057-y.

H. Kitagawa $(\bowtie) \cdot$ T. Ohta

Department of Gastroenterologic Surgery,

Graduate School of Medical Science,

Kanazawa University, 13-1 Takaramachi,

Kanazawa 920-8641, Japan

e-mail: kitagawa@surg2.m.kanazawa-u.ac.jp ligated portion of the main pancreatic duct usually exists at the cut end, and this will become friable with ischemic damage. In some cases, the ligated portion will be broken by ischemic necrosis. The pancreatic fistula from the main pancreatic duct will be more severe than from the small branches. Nonclosure of the cut end of the pancreas contributes to maintaining the blood supply. Therefore, the ligated portion will be stable. We believe that the nonclosure technique resolves the ischemic problem of the main pancreatic duct at the cut end.

The Roux-en-Y end-to-end pancreaticojejunostomy after distal pancreatectomy is available for draining all the interrupted small ductal branches and promoting decompression of the main duct [2]. This method, while theoretically excellent in these areas, is not accepted generally, as it is complicated and not sterilize. Moreover, the failure of anastomosis causes leakage of intestinal juice containing enterokinase and stimulates trypsinogen to trypsin, which is secreted from the pancreas. While fistula from the interrupted small branches occurs occasionally with our method (recently, in 2 out of 25 patients), the drainage placed around the pancreatic cut end resolved this problem.

\section{References}

1. Strasberg SM, Drebin JA, Mokadam NA, Green DW, Jones KL, Ehlers JP, et al. Prospective trial of a blood supply-based technique of pancreaticojejunostomy: effect on anastomotic failure in the Whipple procedure. J Am Coll Surg. 2002;194:746-59.

2. Adam U, Makowiec F, Riediger H, Trzeczak ST, Benz ST, Hopt UT. Distal pancreatic resection: indications, techniques and complications. Zentralbl Chir. 2001;126:908-12. 